\title{
Ultrasonic Properties of Magnetic Nanoparticles with an Additional Biocompatible Dextrane Layer
}

\author{
Leszek DĄBEK, Tomasz HORNOWSKI, Arkadiusz JÓZEFCZAK, Andrzej SKUMIEL \\ Institute of Acoustics, Faculty of Physics, Adam Mickiewicz University \\ Umultowska 85, 61-614 Poznań, Poland; e-mail: hornaku@amu.edu.pl \\ (received October 2, 2012; accepted January 22, 2013)
}

\begin{abstract}
The temperature dependence of the particle size distribution (PSD) of the magnetic fluid with an additional biocompatible dextran layer was studied using a ultrasonic method. The measurements of the ultrasound velocity and attenuation were carried out as a function of the volume concentration of magnetite particles at temperatures ranging from $15^{\circ} \mathrm{C}$ to $40^{\circ} \mathrm{C}$. In order to extract the PSD from ultrasonic measurements, the theoretical model of Vinogradov-Isakovich was used. The extraction of PSD from the ultrasonic data requires also the measurements the density and viscosity of the ferrofluid samples. The calculated PSD of the magnetic fluid with an additional biocompatible layer shows a greater thermal stability than that of a magnetic fluid with a single surfactant layer.
\end{abstract}

Keywords: magnetic nanoparticles, ultrasound, particle size distribution.

\section{Introduction}

According to the usual definition, magnetic nanoparticles can attain a size between $1 \mathrm{~nm}$ and $100 \mathrm{~nm}$. Their importance relates to the fact that the characteristics of the magnetic nanoparticles are different from those of bulk materials of the same composition, which is due mainly to the size effects, magnetic and electronic properties, and the role played by surface phenomena as the size is reduced. Magnetic nanoparticles dispersed in a carrier fluid are called magnetic liquids or ferrofluids. Due to the possibility of remote controlling the parameters of the fluid containing magnetic nanoparticles with the aid of an external magnetic field, magnetic liquids have attracted considerable interest because of potential applications in technological, biological and medical domains, such as seals, bearings, sensors, drug delivery or magnetic hyperthermia.

The parameter that control the distinctive features of magnetic nanoparticles in magnetic fluids is the particle size which determines the scale of interparticles interactions and the concentrations. Real magnetic fluids are always polydisperse systems with a log-normal particle size distribution and a mean magnetic core diameter of about $10 \mathrm{~nm}$ (REGULSKA et al., 2007; RAŞA, 2000).
Since the magnetic moments of the particles are proportional to the volume of their magnetic cores, larger particles can be oriented more easily at low field strengths, and the smaller ones can be oriented only under rather strong magnetic fields applied. As a result, the magnetization properties of a polydisperse system differ from that of a monodisperse system even in the case of very diluted magnetic fluids. More importantly, a small fraction of the large particles in the real system has a typical size of 15-20 nm. Since their dipole-dipole interaction energy is considerably larger than the thermal energy at room temperature, these particles can aggregate into bigger structures. Experiments have demonstrated that this small fraction of large particles can play a major role in determining the physical properties of polydisperse magnetic fluids (BACRI et al., 1990). Aggregation of magnetic particles due to magnetic interactions could block vessels and capillaries when magnetic fluids are used in drugdelivering therapies. Thus prior to the use of a the magnetic fluid in medical therapies it is necessary to study the particle (or aggregate) size distribution (PSD) and its dependence on temperature.

Among the methods commonly used to measure the PSD function and study its properties are ultrasonic techniques, which are recognized as very promising from an industrial point of view (DUKHIN, 
Goetz, 1998). In the so-called ultrasonic spectroscopy (HorNowski et al., 2008), the particle size distribution and concentration of a colloidal dispersion is usually determined by measuring the ultrasonic velocity and/or attenuation coefficient as a function of frequency and using a suitable mathematical model to interpret the spectra. However, it was shown (JóZEFCZAK et al., 2011) that in case of diluted magnetic fluids it is also possible to extract the parameters of the PSD function from the dependence of ultrasonic data measured only at one frequency on the concentration of magnetic nanoparticles.

The purpose of this paper is to compare the PSDs obtained by the ultrasonic method for two types of magnetic fluids with different structures of the stabilizing layer. In order to achieve the repulsive mechanism preventing agglomeration, the particles were coated by a surfactant that produces an entropic repulsion. This constitutes one stabilizing layer surrounding the magnetic core. However, some biomedical applications require an encapsulation of the magnetic core by an additional inorganic or a polymeric coating that renders the particles biocompatible and may serve as a support for biomolecules. The first type of magnetic fluids studied in this work was composed of a single surfactant stabilizing layer, the second one posses an additional biocompatible layer.

\section{Materials and methods}

The superparamagnetic nanoparticles were prepared by co-precipitation using ferric and ferrous salts and ammonium hydroxide. The freshly prepared magnetic nanoparticles (magnetite) were sterically stabilized by sodium oleate ( $c a$. 1:1 to $\mathrm{Fe}_{3} \mathrm{O}_{4}$ ) to prevent their agglomeration. By centrifuging $30 \mathrm{~min}$ at $9000 \mathrm{rpm}$ an initial magnetic fluid (sample 1 - MF, arose. Then, dextran (average molecular weight $=$ 64000 Daltons) was added to achieve a dextran to $\mathrm{Fe}_{3} \mathrm{O}_{4}$ weight ratio equal to 0.5 (sample 2 - MF Dextran) (KonerackÁ et al., 2010). Dextran is a polymer $\left(\mathrm{C}_{6} \mathrm{H}_{10} \mathrm{O}_{5}\right)_{n}$, of anhydroglucose having mainly alpha-D(1-6) linkages with some unusual 1,3 glucosidic linkages at branching points (BAUTISTA et al., 2005).

From the magnetization curve obtained using SQUID, the saturation magnetization and volume concentration, $\phi_{0}$, of the magnetite particles in both samples were determined (RAŞA, 2000, ROZYNEK et al., 2011). Samples with six different volume concentrations of magnetite particles have been prepared by diluting the initial ferrofluid with water. The volume concentration of the samples studied can be found in Table 1. This method of preparation of the samples should not affect the particle sizes as these kinds of ferrofluids have been shown to be stable for long periods. In other words, the measured PSD for the diluted magnetic fluids is independent of the volume concentration of the samples (YoON, 2009).

Table 1. Volume concentration of magnetic material, $\phi_{m}$ for the samples studied.

\begin{tabular}{|c|c|c|c|c|c|c|}
\hline Samples & $\begin{array}{c}\phi_{0} \\
{[\%]}\end{array}$ & $\begin{array}{c}\phi_{1} \\
{[\%]}\end{array}$ & $\begin{array}{c}\phi_{2} \\
{[\%]}\end{array}$ & $\begin{array}{c}\phi_{3} \\
{[\%]}\end{array}$ & $\begin{array}{c}\phi_{4} \\
{[\%]}\end{array}$ & $\begin{array}{c}\phi_{5} \\
{[\%]}\end{array}$ \\
\hline MF & 1.38 & 0.69 & 0.35 & 0.17 & 0.09 & 0.04 \\
\hline MF Dextran & 1.40 & 0.70 & 0.35 & 0.17 & 0.09 & 0.04 \\
\hline
\end{tabular}

The ultrasonic measurements were carried out using the ResoScan (Germany) ultrasonic device, which measures the ultrasonic velocity, $c$, and attenuation, $\alpha f^{-2}$, of the sample, where $f$ is the frequency of the ultrasonic wave. The resolution of the ultrasonic velocity is $0.01 \mathrm{~m} \mathrm{~s}^{-1}$. The relative error of the ultrasonic attenuation coefficient was calculated from the series of repeated measurements and is better than $5 \%$.

The shear viscosity coefficient was measured using a cone-and-plate Brookfield DV II+ viscometer within $10^{\circ} \mathrm{C}$ to $40^{\circ} \mathrm{C}$ temperature range with a temperature control uncertainty of $0.2^{\circ} \mathrm{C}$.

The density was measured using a DMA-38 oscillating U-tube Anton Paar density meter that measures sample density values accurately to $1 \mathrm{~kg} \mathrm{~m}^{-3}$ in the temperature range of $15^{\circ} \mathrm{C}$ to $40^{\circ} \mathrm{C}$.

\section{Results and discussion}

In order to extract the PSD from the ultrasonic data, it was necessary to measure densities and shear viscosities for both the types of magnetic fluids studied in the experiment. The densities of the samples were measured within the temperature range $15^{\circ} \mathrm{C}$ to $40^{\circ} \mathrm{C}$; the results of measurements are shown in Figs. 1 and 2. The variation of the density with temperature for all the samples studied obeys the quadratic relation.

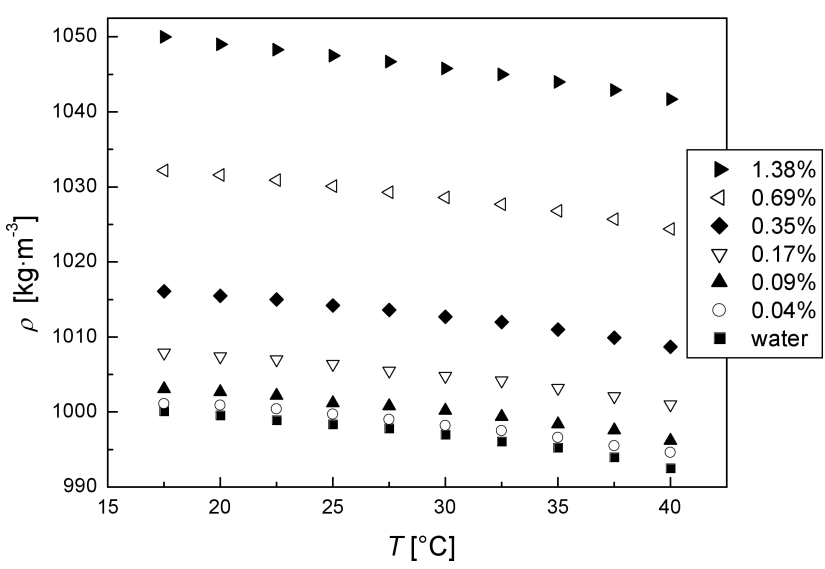

Fig. 1. Temperature dependencies of density of the magnetic fluid with a single surfactant layer (MF) for different concentrations of magnetic nanoparticles. 


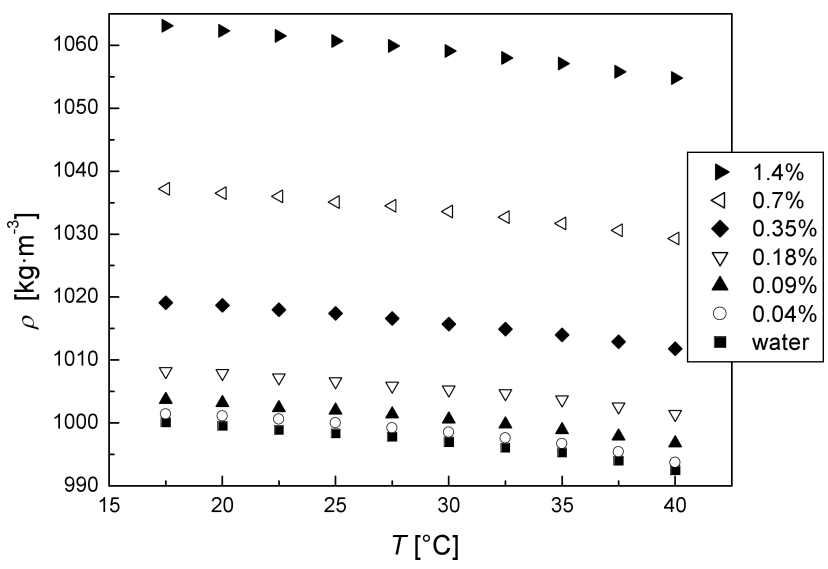

Fig. 2. Temperature dependencies of density of the magnetic fluid with both the surfactant and dextran layers for different concentrations of magnetic nanoparticles.

Figures 3 and 4 show the temperature dependence of the viscosity for both the types of the magnetic fluids

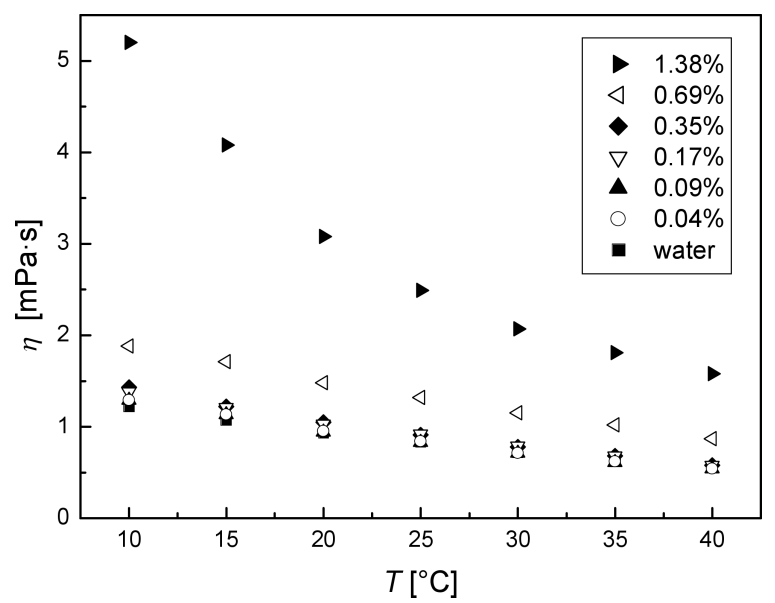

Fig. 3. Temperature dependence of the shear viscosity in magnetic fluids with a single surfactant layer for different concentrations of magnetic nanoparticles.

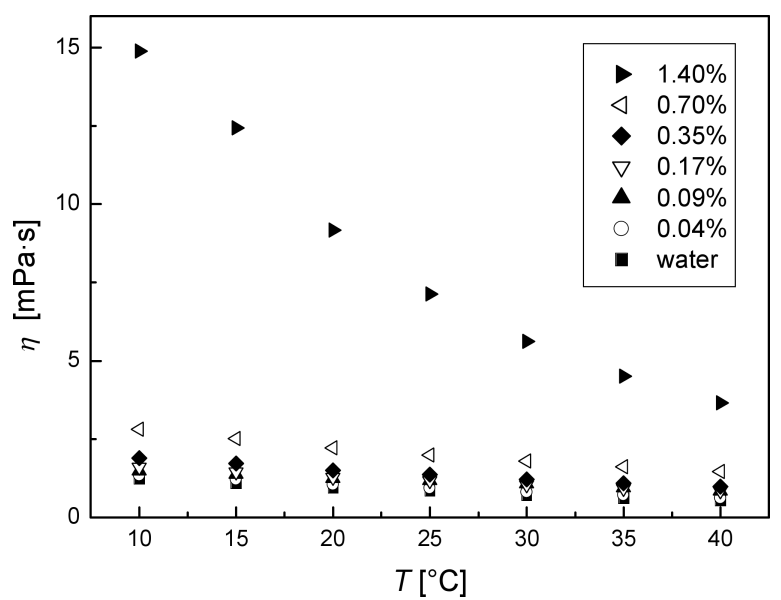

Fig. 4. Temperature dependence of the shear viscosity in magnetic fluids with both surfactant and biocompatible layers for different concentrations of magnetic nanoparticles. studied. The viscosity increases after the addition of dextran and decreases with temperature according to the Arrhenius law (JózEFCZAK et al., 2013).

To calculate the PSD function from ultrasonic attenuation data, information about the various physical parameters of the solid particles and the liquid dispersion medium is required. The studied ferrofluids were considered as aggregates composed of magnetite/oleic acid in the case of the magnetic fluid with a single surfactant layer and as magnetite/oleic acid+dextran in the case of the magnetic fluid with both surfactant and biocompatible shells. It was assumed that these aggregates are spherical and dispersed in water. The aggregates densities, the oleic acid and dextran volume concentrations can be calculated from the physicochemical model described in details in (HoRNOwsKI et al., 2008; JózEFCZAK et al., 2011).

The results of the ultrasonic velocity measurements are shown in Figs. 5 and 6 . The results obtained are in

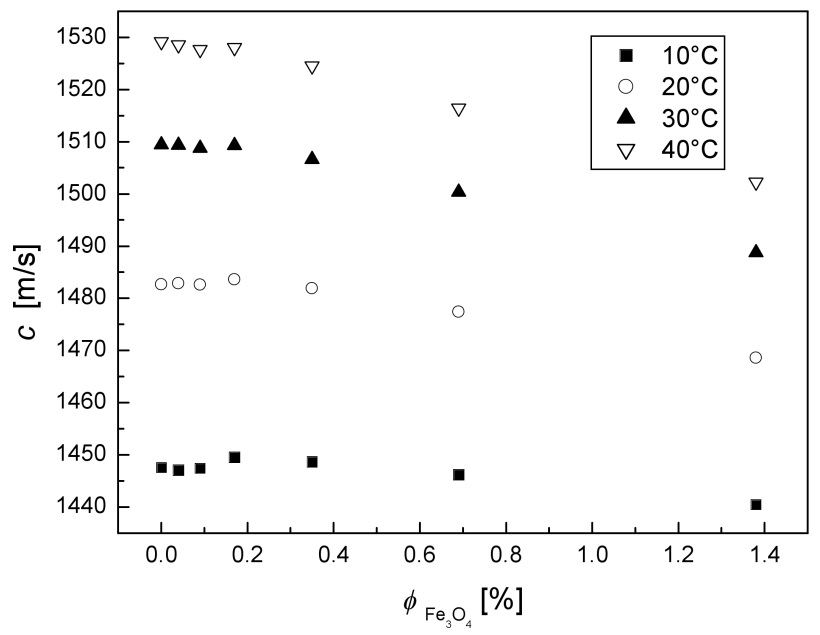

Fig. 5. Ultrasonic wave velocity as a function of the magnetite volume concentration in magnetic fluids with a single surfactant layer for different temperatures.

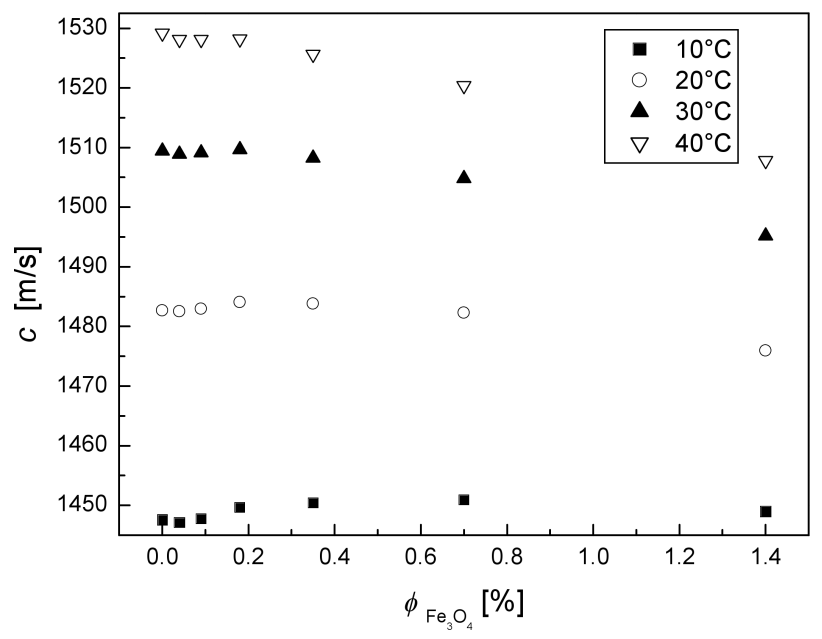

Fig. 6. Ultrasonic wave velocity as a function of magnetite volume concentration in magnetic fluids with both surfactant and biocompatible layers for different temperatures. 
a qualitative agreement with simple macroscopic theories that predict a parabolic relationship between the ultrasonic velocity in a colloidal mixture and the volume concentration of the continuous phase (CHALLIS et al., 2005).

Figures 7 and 8 shows the experimental results of ultrasonic attenuation, $\alpha f^{-2}$, as a function of the magnetite particle concentration for temperature range of $10^{\circ} \mathrm{C}$ to $40^{\circ} \mathrm{C}$. For all the temperatures the ultrasonic attenuation increases with increasing volume concentration of the magnetite particles.

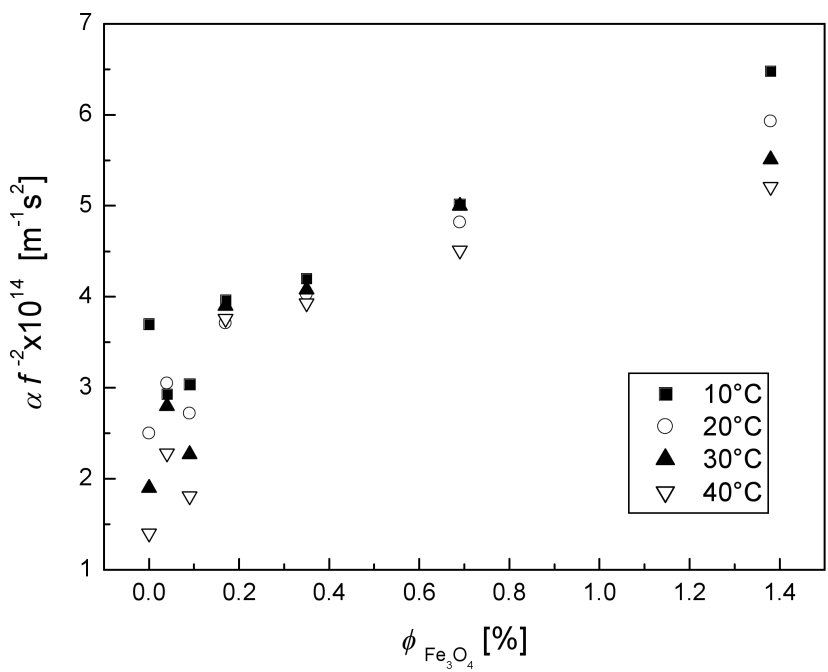

Fig. 7. Ultrasonic wave attenuation as a function of the magnetite volume concentration in magnetic fluids with a single surfactant layer for different temperatures.

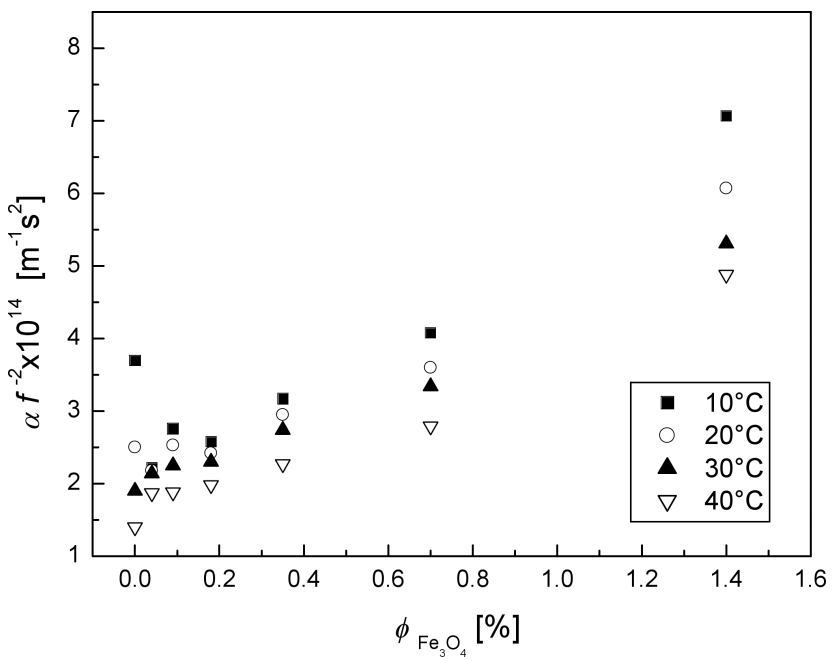

Fig. 8. Ultrasonic wave attenuation as a function of magnetite volume concentration in magnetic fluids with both surfactant and biocompatible layers for different temperatures.

The interaction between the acoustic wave and the suspended particles of nanometer size leads to an additional attenuation of the sound compared to that in the carrier liquid, $\alpha_{0}$. This additional term $\Delta \alpha$ describes the attenuation that can be attributed to visco-inertial, thermal, and scattering losses (DUKHIN, Goetz, 2001). The additional attenuation depends on the particle size distribution that can be assumed to obey a log-normal distribution characterized by the density as a sum of the absorption coefficient of dispersion:

$$
\begin{aligned}
p(R)= & \frac{1}{\sqrt{2 \pi} \sigma\left(R-R_{0}\right)} \\
& \cdot \exp \left(-\frac{\left[\ln \left(R-R_{0}\right)-m\right]^{2}}{2 \sigma^{2}}\right) .
\end{aligned}
$$

Here $m$ is the logarithmic mean, $\sigma$ is the logarithmic standard deviation, and $R_{0}$ is a minimal radius. The mean $m$ and standard deviation $\sigma$ which give the best agreement between the measured and predicted ultrasonic attenuation can be found by a least squares analysis.

The mean value $M_{\phi}$, and root-mean-square deviation $D_{\phi}$, of the particle radius $R$ can be determined from the expressions:

$$
\begin{aligned}
& M=\exp \left(m+0.5 \sigma^{2}\right)+R_{0}, \\
& D=\exp \left(2 m+\sigma^{2}\right)(\exp \sigma-1) .
\end{aligned}
$$

To extract the parameters of the particle size distribution from ultrasonic attenuation data, the theoretical model proposed by Vinogradov and Isakovich (VINOGRADOV, 2003) was used.

In this model three mechanisms are responsible for the additional ultrasound attenuation in ferrofluids with respect to that of the carrier liquid: viscoinertial absorption, $\alpha_{\eta}$, associated with the difference in density between the magnetic nanoparticles and the carrier liquid, thermal absorption, $\alpha_{T}$, associated with the difference in the thermal properties between the magnetic particles and the carrier liquid and scattering losses, $\alpha_{S}$, associated with the difference in the acoustic impedance. Neglecting the scattering effect which is very small for the wave with frequency equal to $8 \mathrm{MHz}$, the overall ultrasonic attenuation in the magnetic liquid per frequency squared can be conveniently expressed as the sum of the first two contributions:

$$
\frac{\alpha}{f^{2}}=\frac{\alpha_{\eta}}{f^{2}}+\frac{\alpha_{T}}{f^{2}} .
$$

The contributions to the attenuation of sound due to viscoinertial and thermal processes are given by

$$
\begin{aligned}
\frac{\alpha_{\eta}}{f^{2}}= & \frac{K \pi \phi}{c} \int_{R_{\min }}^{R_{\max }} f(R) R^{2} \\
& \times \frac{Q(R, f)+S(R, f) q_{3} \tau_{V} f}{Q(R, f)^{2}+W(R, f)} \mathrm{d} R+\frac{2 \pi^{2} \tau_{V}}{c},
\end{aligned}
$$

where

$$
K=\frac{4 \pi(1-\phi)\left(\rho_{a}-\rho_{f}\right)^{2}}{9 \rho \eta_{f}}, \quad Q(R, f)=1+R \sqrt{q_{1} f},
$$




$$
\begin{aligned}
& S(R, f)=R \sqrt{q_{1} f}+\frac{2}{9} q_{1} R^{2} f+q_{2} R^{2} f \\
& W(R, f)=q_{1} R^{2} f+\frac{4}{9} R^{3} \sqrt{\left(q_{1} f\right)^{3}}+2 q_{2} R^{3} \sqrt{q_{1} f}, \\
& q_{1}=\frac{\pi \rho_{f}}{\eta_{f}}, \quad q_{2}=\frac{4 \pi(1-\phi) \rho_{a} \rho_{f}}{9 \rho \eta_{f}}, \\
& q_{3}=\frac{4 \pi \rho_{a}^{2}}{(1-\phi)\left(\rho_{a}-\rho_{f}\right)^{2}}, \quad \tau_{V}=\frac{\alpha_{f}}{f^{2}} \frac{\rho_{f} c_{f}^{3}}{2 \pi^{2} \rho c^{2}}, \\
& \frac{\alpha_{T}}{f^{2}}=-\frac{\phi}{f^{2}} \int_{R_{\min }}^{R_{\max }} f(R) \\
& \cdot \operatorname{Im}\left[-\frac{j \lambda_{a} \lambda_{f}\left[Z_{a}-\tanh \left(Z_{a}\right)\right]\left(Z_{f}+1\right)}{\lambda_{a}\left[Z_{a}-\tanh \left(Z_{a}\right)\right]+\lambda_{f}\left(Z_{f}+1\right) \tanh \left(Z_{a}\right)}\right] \mathrm{d} R, \\
& Z=\frac{3 \rho T c}{2 R^{2}}\left(\frac{\gamma_{a}}{\rho_{a} C_{a}}-\frac{\gamma_{f}}{\rho_{f} C_{f}}\right)^{2}, \\
& Z_{a}=(1+j) R \sqrt{\pi f \rho_{a} C_{a} \lambda_{a}^{-1}}, \quad j=\sqrt{-1}, \\
& Z_{f}=(1+j) R \sqrt{\pi f \rho_{f} C_{f} \lambda_{f}^{-1}} .
\end{aligned}
$$

Here $\rho, \beta, \eta, c$ and $\alpha$ are the density, adiabatic compressibility, viscosity, velocity and sound absorption, $T$ is the temperature of the medium, and $C, \lambda$, and $\gamma$ are the heat capacity at constant pressure, the heat conductivity coefficient and the thermal expansion coefficient. The subscripts $a$ and $f$ refer to the properties of the magnetite particles and the continuous phase, respectively.

Figures 9 and 10 show the log-normal PSDs obtained by the ultrasonic method for various temperatures in the range of $10^{\circ} \mathrm{C}$ to $40^{\circ} \mathrm{C}$.

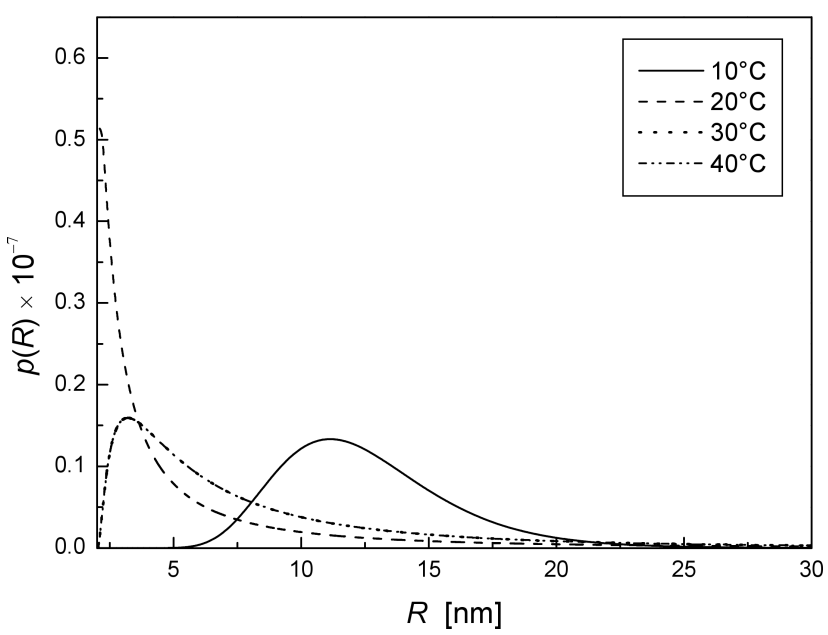

Fig. 9. Log-normal PSD functions in magnetic fluids with a single surfactant layer obtained from ultrasonic measurements for different temperatures.

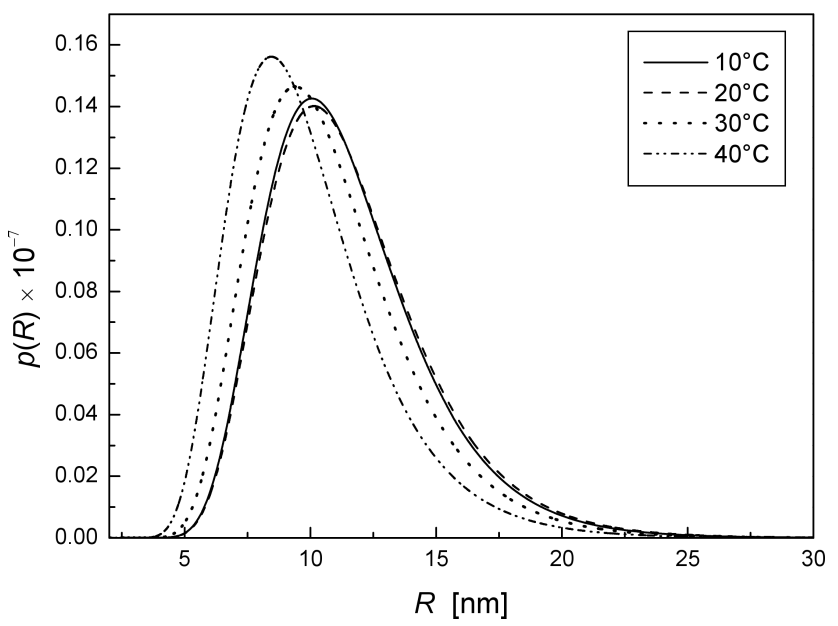

Fig. 10. Log-normal PSD functions in magnetic fluids with both the surfactant and biocompatible layers obtained from ultrasonic measurements for different temperatures.

Figures 9 and 10 present PSD functions extracted from the ultrasonic attenuation data for both the types of magnetic fluids studied in the experiment. The values of the PSD function parameters, $m$ and $\sigma$, as well as the values of $M_{\phi}$ and $D_{\phi}$ for all temperatures are listed in Table 2. The mean particle radius $M_{\phi}$, evaluated from ultrasonic measurements is bigger by twice as much as the average magnetic nanoparticle size. This is because the ultrasonic granulometry gives the value of the so-called "hydrodynamic" radius which is greater than the size of the magnetic core by a magnitude, $\delta_{s}+\delta_{m}$, where $\delta_{s}$ denotes the thickness of the protective surfactant layer/layers and $\delta_{m}$ is the thickness of the magnetically inactive layer on the surface of the particles (Pshenichnikov et al., 1996).

Table 2. Parameters $m$ and $\sigma$ of the log-normal particle size distribution, mean value $M_{\phi}$, and root-mean-square deviation $D_{\phi}$ of particle radius $R$ calculated from ultrasonic data for different temperatures.

\begin{tabular}{|c|c|c|c|c|c|}
\hline \multirow{3}{*}{ Sample } & $\begin{array}{c}T \\
{\left[{ }^{\circ} \mathrm{C}\right]}\end{array}$ & $-m$ & $\sigma$ & $\begin{array}{c}M_{\phi} \times 10^{9} \\
{[\mathrm{~m}]}\end{array}$ & $\begin{array}{c}D_{\phi} \times 10^{18} \\
{\left[\mathrm{~m}^{2}\right]}\end{array}$ \\
\hline \multirow{5}{*}{$\mathrm{MF}$} & 10 & 18.414 & 0.312 & 12.57 & 40.90 \\
\cline { 2 - 6 } & 20 & 20.194 & 1.590 & 8.01 & 140.10 \\
\cline { 2 - 6 } & 30 & 19.292 & 1.118 & 9.82 & 125.78 \\
\cline { 2 - 6 } & 40 & 18.947 & 0.857 & 10.53 & 125.78 \\
\hline \multirow{5}{*}{ MF+Dextran } & 10 & 18.529 & 0.329 & 11.47 & 34.95 \\
\cline { 2 - 6 } & 20 & 18.517 & 0.331 & 11.59 & 36.11 \\
\cline { 2 - 6 } & 30 & 18.603 & 0.347 & 10.85 & 32.49 \\
\cline { 2 - 6 } & 40 & 18.723 & 0.370 & 9.91 & 28.05 \\
\hline
\end{tabular}

The PSDs of magnetic fluid with a single surfactant layer shown in Fig. 9 change substantially with temperature. This instability can be related to the creation and destruction of magnetic particle aggregates and is confirmed by rather large values of the root- 
mean-square deviation $D_{\phi}$ of the particle radius. However, at human body temperature this process seems to run slower. The addition of the dextran layer has a dramatic impact on the magnetic fluid PSD as can be seen in Fig. 10. The PSDs show now an excellent stability for all temperatures. This proves that apart from providing additional biocompatibility, the dextran layer can also prevent agglomeration of magnetic nanoparticles.

\section{Conclusions}

The effect of a dextran additional layer on the particle size distribution was studied. The ultrasonically determined PSD functions show that the surface modification of magnetic nanoparticles with dextran enhances the stability of biocompatible magnetic fluids. This finding is in agreement with our studies of the rheological properties of dextran modified magnetic nanopaticles (JózEFCZAK et al., 2013).

\section{Acknowledgment}

This work was supported by the Polish National Science Centre grant number DEC-2011/03/B/ST7/00194. The authors gratefully acknowledge Professor Milan Timko from the Slovak Academy of Science (Košice) for samples of the magnetic fluids.

\section{References}

1. Bacri J. C., Perzynski R., SAlin D. (1990), Ionic ferrofluids: A crossing of chemistry and physics, J. Magn. Magn. Mater., 85, 27-32.

2. Bautista M.C., Bomati-Miguel O., Morales M.P., Serna C.J., Veintemillas-Verdaguer S. (2005), Surface characterisation of dextran-coated iron oxide nanoparticles prepared by laser pyrolysis and coprecipitation, J. Magn. Magn. Matter., 293, 20-27.

3. Challis R. E., Povey M. J., Mather M. L., HolMES A. K. (2005), Ultrasound techniques for characterizing colloidal dispersions, Rep. Prog. Phys., 68, 15411637.

4. Dukhin A.S., Goetz P. J. (1998), Characterization of aggregation phenomena by means of acoustic and electroacoustic spectroscopy, Colloids Surf. A, 144, 4958.
5. Dukhin A.S., Goetz P. J. (2001), Acoustic and electroacoustic spectroscopy for characterizing concentrated dispersions and emulsions, Adv. Colloid Interface Sci., 92, 73-132.

6. Hornowski T., Józefczak A., Łabowski M., SkuMIEL A. (2008), Ultrasonic determination of the particle size distribution in water-based magnetic liquid, Ultrasonics, 48, 594-597.

7. Józefczak A., Hornowski T., Rozynek Z., SkuMIEL A., Fossum J. O. (2013), Rheological study of dextran modified magnetite nanoparticles water suspension, Int. J. Thermophys., to be published.

8. Józefczak A., Hornowski T., Skumiel A. (2011), Temperature dependence of particle size distribution in transformer oil-based ferrofluid, Int. J. Thermophys., 32, 795-806.

9. Koneracká M., Antošová A., ZÁvišová V., LanCZA G., GaŽová Z., ŠIpošová K., Juríková A., Csach K., KováC J., Tomašovicová N., FABiÁn M., KopcanskÝ P. (2010), Characterization of $\mathrm{Fe}_{3} \mathrm{O}_{4}$ Magnetic Nanoparticles Modified with Dextran and Investigation of Their Interaction with Protein Amyloid Aggregates, Acta Phys. Pol. A, 118, 983-985.

10. Pshenichnikov A.F., Mekhonoshin V. V., LebeDEV A.V. (1996), Magneto-granulometric analysis of concentrated ferrocolloids, J. Magn. Magn. Mater., 161, 94-102.

11. RAŞA M. (2000), Magnetic properties and magnetobirefringence of magnetic fluids, Eur. Phys. J. E, 2, 265-275.

12. Regulska P., Skumiel A., Hornowski T., JózefCZAK A. (2007), Magnetic properties and anisotropy of ultrasound attenuation in APG-832 magnetic liquid, Archives of Acoustics, 32, 4 (Supplement), 95-100.

13. Rozynek Z., Józefczak A., Knudsen K. D., Skumiel A., Hornowski T., Fossum O., Timko M., KopČAnský P., KonerackÁ M. (2011), Structuring from nanoparticles in oil-based ferrofluids, Eur. Phys. J. E, 34, 28.

14. Vinogradov A. N. (2003), Application of Acoustic Spectroscopy to Investigation of Microinhomogeneous Media, Colloid J., 65, 539-544.

15. Yoon S. (2009), Magnetic Methods for Determining the Monodispersity of Ferrouids, J. Korean Phys. Soc., 54, 163-168. 